\title{
REPORT
}

\section{EXPERIENCES IN A LEVEL-1 TRAUMA CENTRE}

In September 1991 I spent four weeks acting as an intern at a level one trauma centre in the United States. An Advanced Trauma Life Support (ATLS) course was attended leading to certification from the American College of Surgeons. Its protocol was carried out on over 80 major trauma patients.

The University Medical Centre in downtown Jacksonville, Florida came as a culture shock. There was a constant awareness of the high incidence of violence that pervades the surrounding area. The deserted pavements, abandoned shop premises and the presence of armed police on duty within hotel lobbies were all signs of the troubles that beset this city.

Warnings were repeatedly given not to walk around in the city centre by day or night. The buddy buddy system was advised when walking in the hospital grounds between accommodation and the main hospital or alternatively to call security for an escort. Perhaps the most poignant and forceful argument to exercise a degree of caution was provided by a visit to the emergency room (ER).

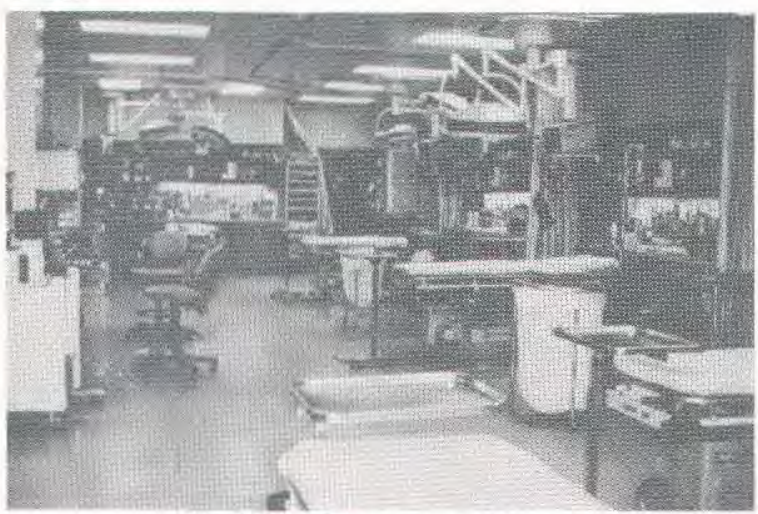

Fig 1. The Emergency Room.

This room has six bays each fully equipped for resuscitation (Fig 1). It is one of four level one trauma centres in Florida and in addition also receives level two and level three patients as defined by standard criteria (Fig 2). On my initial tour a couple of patients were being resuscitated. A black male in his early twenties had been shot twice in the left side of his face leaving two clearly defined entrance wounds each of about $3 \mathrm{~cm}$ in diameter. No exit wounds were evident. Efforts at resuscitation ceased once skull views were available (Fig 3 ). Resuscitation included a thoracotomy with clamping of the thoracic aorta, repair of the atrial wound and open heart massage. All procedures were carried out as a matter of routine and within the ER.

About 240 major cases are received by the trauma service each month made up largely of road traffic accidents (RTAs), gunshot wounds (GSWs) and stab
Level one trauma: (Rule of 9's)

Glasgow coma scale of 9 or less

Systolic blood pressure of 90 or less

Respiratory rate of 9 or less

\section{Level two trauma:}

Glasgow coma scale of 12 or less

Respiratory rate less than 10 or greater than 29

Penetrating injury to head/neck/chest/back abdomen or groin

2nd or 3rd degree burns involving less than $15 \%$

surface area

Paralysis

Amputation proximal to wrist or ankle

Ejection from motor vehicle

\section{Level three trauma:}

Multiple trauma with burns greater than $10 \%$

Limb paralysis

Death in same passenger compartment

Extrication time greater than 10 minutes

Pelvic fracture

Flail chest

2 or more proximal long bone fractures

Fig 2. Criteria for designating trauma level.

wounds (SWs) (Fig 4). With a one in three on call ifico possible to be involved in over 80 resuscitations durgig the month. On one Saturday night we received 6 GS 3 SWs and 5 RTAs. There were up to 60 major trau cases on the trauma wards which had not been trañs ferred to another speciality. There were quiet days an@ they were the ones which excited comment. Experienco was gained in all aspects of assessment and resuscitatio and carrying out emergency operative procedures. Tho latter were all initially carried out in the dog laboratory

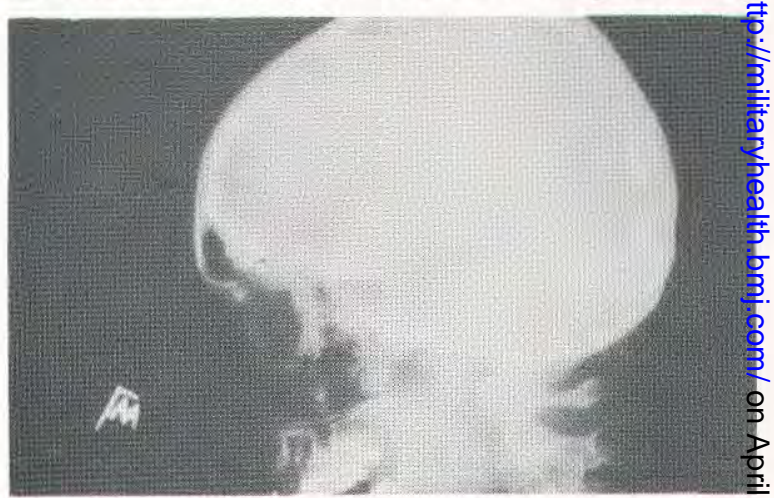

Fig 3. Lateral skull radiograph following two gunshos wounds for a low velocity weapon to the left side of the face. 


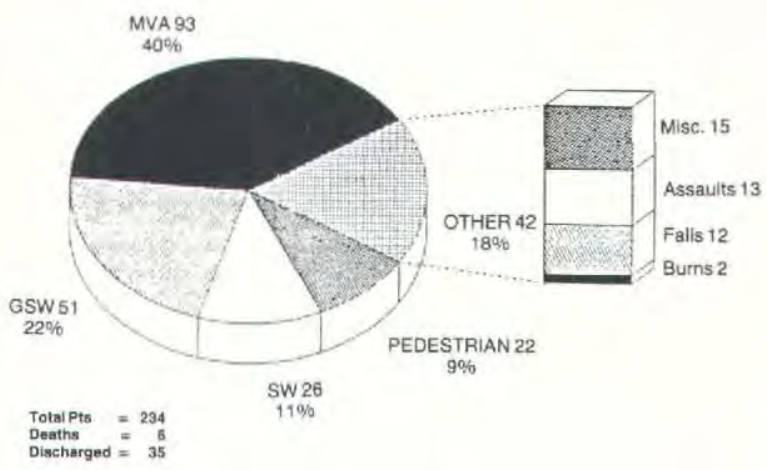

Fig 4. A monthly audit of trauma patients received.

including pericardiocentesis and diagnostic peritoneal lavage as part of the ATLS course. The consistent high volume of trauma is such that there is a wealth of experience to be gained, Medical indemnity was covered by the hospital at Jacksonville.

Patient transportation to the hospital ranged from the orthodox to the somewhat bizarre. The former included a BK 117 twin engined helicopter (Fig 5) which covers a 120 mile radius and is permanently on standby together with pilots, nurses, mechanics and control staff. The helicopter "Trauma One" can carry two patients and is in constant contact with the control room which is colocated with the ER. Advance information regarding the patient may be received over the radio and in turn instructions such as the use of restricted drugs may be given by the duty physician to the on-board nurse. Patients arriving via this facility would have been managed using the ATLS protocol. Procedures facility would have been managed using the ATLS protocol. Procedures such as intubation, insertion of chest tube

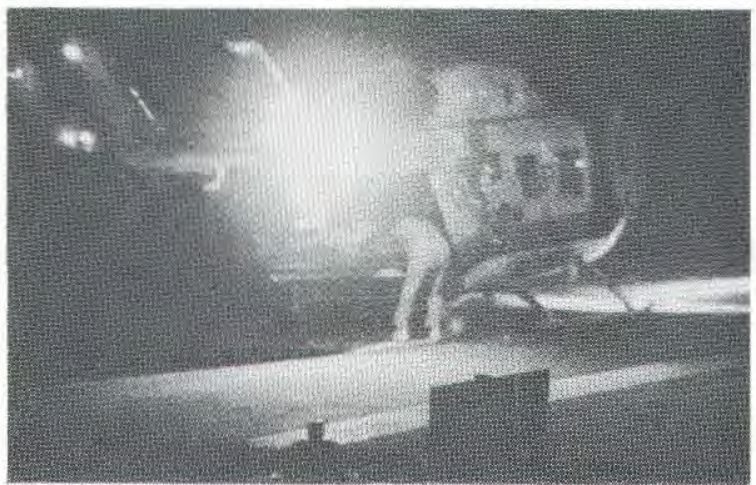

Fig 5. Traumaone: The BK 117 twin engined helicopter used for patient transfer. and the initiation of IV infusions could all be carried ou? prior to arrival at the ER. Splinting equipment an MAST trousers were also available and frequentl $\mathbf{z}$ utilised.

Less orthodox was the back seat of a friend's car to bring in a friend or relative rapidly exsanguinating fron one or more fatal GSWs. I witnessed some mos?? extraordinary sights. In one of many such incidents black youth aged twenty years came frantically running into the ER with arms wildly gesticulating and pleadin urgently for a doctor to help his cousin who had bee핟․ shot. With no prior warning of his arrival the traum service was rapidly mobilised. The trauma nurse quickly followed him outside to reappear with a trolleøै upon which was a patient straddled by a nurse giving cardiac massage whilst blood poured from his mouth: This rare and understandable breach of protocol was. followed by intubation and thoracotomy. Augmentatios of the IV infusions via venous cutdowns of his left an@ right long saphenous veins was from a Foley cathete inserted into his right atrium.

More bizarre still would be the fatally wounded walk ing their last steps unaided into the ER accompanied by noisy and angry mob. Each member of the mob wo consider themselves to be indispensable with regarce the resuscitation of the patient. The crescendo of nowe would be increased as the unarmed trauma team wo demand the exit of the mob leaving space and tranquifiti to resuscitate the patient. When initial woundings ropo have occurred as a result of an altercation over a dollars it is not surprising that in this frenzied and highlv charged atmosphere further utilisation of firearms mighto be deemed appropriate. ER shootings do occur altho none to date have occurred at Jacksonville. Never less, following one such incident and an unfavourable cranial CT scan there was a certain reticence on the paro of individual members of the trauma team to volunteeब to approach the would-be helpers regarding the thorn question of organ donation.

The experience to be gained in one month at a leveltrauma centre in the United States is recommended.

MAjor R PILCHER, BSc, FDSRCPS, RAD

University College and

Middlesex School of Medicine, London SW1

\section{Acknowledgements}

I thank Dr W F Fallon Chief, Division of Traumg Surgery, University Medical Centre, Jacksonville and Dळ $\mathrm{K}$ Sinclair formerly chief resident in trauma Jacksonville for their help. 